\title{
The chromatic number of a signed graph
}

\author{
Edita Máčajová \\ Department of Computer Science \\ Comenius University \\ Mlynská dolina, 84248 Bratislava, Slovakia \\ macajova@dcs.fmph. uniba.sk \\ André Raspaud \\ LaBRI \\ Université de Bordeaux \\ 33405 Talence Cedex, France \\ raspud@labri.fr \\ Martin Škoviera \\ Department of Computer Science \\ Comenius University \\ Mlynská dolina, 84248 Bratislava, Slovakia \\ skoviera@dcs.fmph.uniba.sk
}

Submitted: Dec 24, 2014; Accepted: Jan 11, 2016; Published: Jan 22, 2016

Mathematics Subject Classifications: 05C15, 05C22

\begin{abstract}
In 1982, Zaslavsky introduced the concept of a proper vertex colouring of a signed graph $G$ as a mapping $\phi: V(G) \rightarrow \mathbb{Z}$ such that for any two adjacent vertices $u$ and $v$ the colour $\phi(u)$ is different from the colour $\sigma(u v) \phi(v)$, where is $\sigma(u v)$ is the sign of the edge $u v$. The substantial part of Zaslavsky's research concentrated on polynomial invariants related to signed graph colourings rather than on the behaviour of colourings of individual signed graphs. We continue the study of signed graph colourings by proposing the definition of a chromatic number for signed graphs which provides a natural extension of the chromatic number of an unsigned graph. We establish the basic properties of this invariant, provide bounds in terms of the chromatic number of the underlying unsigned graph, investigate the chromatic number of signed planar graphs, and prove an extension of the celebrated Brooks' theorem to signed graphs.
\end{abstract}

\section{Introduction}

This paper continues the study of vertex colourings of signed graphs initiated by Zaslavsky in three seminal papers $[9,10,11]$ from early 1980's. A signed graph is a graph in which each edge is labelled with +1 or -1 . The idea of how to colour such a graph is fairly straightforward: (1) use signed colours so as to enable vertex-switching, and (2) do so in such a way that the usual rule to colour adjacent vertices with different colours is respected as long as the edge that joins them is positive. The following definition, taken 
from [9], incorporates this idea: Given a signed graph $G$, a proper vertex colouring of $G$, or simply a colouring, is a mapping $\phi: V(G) \rightarrow \mathbb{Z}$ such that for each edge $e=u v$ of $G$ the colour $\phi(u)$ is distinct from the colour $\sigma(e) \phi(v)$, where $\sigma(e)$ denotes the sign of $e$. In other words, the colours of vertices joined by a positive edge must not coincide while those joined by a negative edge must not be opposite.

This definition is natural for several reasons extensively discussed in $[9,10,11]$. In particular, colourings defined in this manner are well behaved under switching. Recall that switching of a signed graph at a vertex $v$ reverses the sign of each non-loop edge incident with $v$. The switching operation does not essentially change the signed graph, because it preserves the sign product on each circuit. If we switch a properly coloured signed graph at some vertex, the colouring has to be switched together with the signature, that is to say, the colour at the vertex must be replaced with its negative. It is easy to see that the result of a vertex switching is again a proper colouring. Furthermore, a colouring of a balanced signed graph, one where the sign product on each simple circuit is positive, exactly corresponds to the usual vertex colouring of the underlying unsigned graph.

Our paper focuses on measuring the complexity of a signed graph colouring by means of a chromatic number. One possible approach to introducing the chromatic number of a signed graphs was proposed by Zaslavsky in [9, p. 218] where a signed colouring of a signed graph $G$ in $k$ colours, or in $2 k+1$ signed colours, is defined to be a mapping $V(G) \rightarrow\{-k,-(k-1), \ldots, 0 \ldots, k-1, k\}$; a colouring is zero-free if it never assumes the value 0 . The necessity of treating zero-free colourings separately derives from a different behaviour of switching with respect to vertices coloured zero. This led Zaslavsky [9, p. 219] to define the chromatic polynomial $\chi_{G}(\lambda)$ of a signed graph $G$ to be the function defined for odd positive arguments $\lambda=2 k+1$ whose value equals the number of proper signed colourings of $G$ in $k$ colours. The balanced chromatic polynomial $\chi_{G}^{\mathrm{b}}(\lambda)$, defined for even positive arguments $\lambda=2 k$, is the function which counts the zero-free proper signed colourings in $k$ colours. Finally, the chromatic number $\gamma(G)$ of $G$, according to Zaslavsky $\left[10\right.$, p. 290], is the smallest nonnegative integer $k$ for which $\chi_{G}(2 k+1)>0$, and the strict chromatic number $\gamma^{*}(G)$ of $G$ is the smallest nonnegative integer $k$ such that $\chi_{G}^{\mathrm{b}}(2 k)>0$.

The disadvantage of Zaslavsky's definitions is that neither of these two varieties of chromatic number is a direct extension of the usual chromatic number of an unsigned graph. This is because, roughly speaking, the chromatic numbers $\gamma$ and $\gamma^{*}$ only count the absolute values of colours. However, it seems natural to require a signed version of any graph invariant to agree with its original unsigned version on balanced signed graphs. We therefore diverge from the definitions adopted by Zaslavsky in $[9,10,11]$ and propose the following.

First, we define, for each $n \geqslant 1$, a set $M_{n} \subseteq \mathbb{Z}$ by setting $M_{n}=\{ \pm 1, \pm 2, \ldots, \pm k\}$ if $n=2 k$, and $M_{n}=\{0, \pm 1, \pm 2, \ldots, \pm k\}$ if $n=2 k+1$. A proper colouring of a signed graph $G$ that uses colours from $M_{n}$ will be called an $n$-colouring. Thus, an $n$-colouring of a signed graph uses at most $n$ distinct colours. Note that if $G$ admits an $n$-colouring, then it also admits an $m$-colouring for each $m \geqslant n$. The smallest $n$ such that $G$ admits an $n$-colouring will be called the chromatic number of $G$ and will be denoted by $\chi(G)$. It is easy to see that the chromatic number of a balanced signed graph coincides with the 
chromatic number of its underlying unsigned graph. Moreover,

$$
\chi(G)=\gamma(G)+\gamma^{*}(G) .
$$

The aim of this paper is to prove several basic results concerning the chromatic number of a signed graph. Our results are divided into three sections. In Section 2 we investigate relationships between the chromatic number of a signed graph and various graph invariants. Among other things, we present bounds on the chromatic number of a signed graph by means of the chromatic number, the acyclic chromatic number, and the arboricity of the underlying unsigned graph. Our main result is a Brooks-type theorem for signed graphs, which will be proved in Section 3. The theorem states that the chromatic number of every simple signed graph $G$ is bounded by $\Delta(G)$, the maximum degree of $G$, unless $G$ is a balanced complete graph, a balanced odd circuit, or an unbalanced even circuit. Finally, in Section 4, we deal with the chromatic number of planar signed graphs. We prove that the chromatic number of every simple signed planar graph is at most 5 and make a conjecture that this bound can be improved to 4 .

\section{Basic properties of signed chromatic number}

We assume that the reader is familiar with the basic concepts of signed graph theory such as balance, switching, switching equivalence, etc. For more information about signed graphs we refer the reader to [8] or to $[9,10,11]$.

Recall that a colouring of a signed graph $G$ is a function $\phi: V(G) \rightarrow \mathbb{Z}$ such that for each edge $e=u v$ of $G$ the colour $\phi(u)$ is distinct from the colour $\sigma(e) \phi(v)$, where $\sigma(e)$ is the sign of $e$. It follows that in order for $G$ to admit a proper colouring, $G$ cannot have a positive loop. Throughout the rest of this paper we therefore forbid positive loops in all our signed graphs. Negative loops and parallel edges are not excluded, nevertheless, a negative loop at a vertex $v$ forbids $\phi(v)=0$, and a pair of differently signed parallel edges between vertices $u$ and $v$ forbids $|\phi(u)|=|\phi(v)|$. In general, colour 0 has a different behaviour from other colours because $-0=0$. Somewhat surprisingly, however, colourings of simple signed graphs behave in a very much similar way as colourings of unsigned graphs.

Before proceeding further we would like to remark that throughout the paper the following argument will be used without mention: if a signed graph resulting from a series of switchings is $n$-colourable, then so is the original signed graph.

We start our investigation by comparing the chromatic number of a signed graph to the chromatic number of its underlying graph. In order to make a clear distinction between a signed graph and its underlying graph, we will use $\underline{G}$ to denote the underlying graph of a signed graph $G$.

Theorem 1. For every loopless signed graph $G$ we have

$$
\chi(G) \leqslant 2 \chi(\underline{G})-1 .
$$

Furthermore, this bound is sharp. 
Proof. Every colouring of $\underline{G}$ with colours in the set $\{0,1, \ldots, n-1\}$ is also a signed colouring of $G$ irrespectively of the signature. For $n=\chi(\underline{G})$ we get a signed colouring of $G$ with $2 \chi(\underline{G})-1$ colours from the set $\{0, \pm 1, \ldots, \pm(n-1)\}=M_{2 n-1}$, implying the required inequality.

For the second part, we display an infinite sequence $\left(G_{n}\right)_{n \geqslant 1}$ of signed graphs such that $\chi(G)=2 \chi(\underline{G})-1$. To construct $G_{n}$, we take one all-positive copy $H_{1}$ of $K_{n}$ and $n-1$ all-negative copies $H_{2}, H_{3}, \ldots, H_{n}$ of $K_{n}$. For $1 \leqslant i \leqslant n$ we denote the vertices of $H_{i}$ by $v_{i, 1}, v_{i, 2}, \ldots, v_{i, n}$ and call any two vertices $v_{i, k}$ and $v_{j, k}$ from different subgraphs $H_{i}$ and $H_{j}$ corresponding. To finish the construction, we insert a positive edge between any pair of non-corresponding vertices from different copies of $K_{n}$.

Observe that the assignment $\phi\left(v_{i, j}\right)=j$ for each $i, j \in\{1,2, \ldots, n\}$ defines a proper colouring of $\underline{G}_{n}$. This colouring is optimal since $\underline{G_{n}}$ contains a copy of $K_{n}$. Hence $\chi\left(\underline{G_{n}}\right)=$ $n$, and by the first part of the proof, $\chi\left(G_{n}\right) \leqslant \overline{2 n}-1$. We now show that the chromatic number of $G_{n}$ is $2 n-1$. Assume, to the contrary, that $G_{n}$ has a colouring with colours from the set $M_{2 n-2}$. Since $H_{1}$ is a balanced complete graph on $n$ vertices, $n$ different colours $c_{1}, c_{2}, \ldots, c_{n}$ have to be used for the vertices of $H_{1}$. We may assume that the vertex $v_{1, i}$ has colour $c_{i}$. Let $D=\left\{d_{1}, d_{2}, \ldots, d_{n-2}\right\}$ be the set of all remaining colours, so that $M_{2 n-2}=\left\{c_{1}, c_{2}, \ldots, c_{n}, d_{1}, d_{2}, \ldots, d_{n-2}\right\}$.

There are $n$ vertices in each $H_{i}$ with $i \geqslant 2$ but only $n-1$ distinct absolute values in $M_{2 n-2}$, so there must be two vertices in each such $H_{i}$ that receive colours with the same absolute value. Since the edges within each $H_{i}$ with $i \geqslant 2$ are all negative, such a pair of vertices must receive the same colour. Further, any vertex $v_{i, j}$ of $H_{i}$ with $i \geqslant 2$ receives a colour from the set $\left\{c_{j}\right\} \cup D$. It follows that the repeated colour in each $H_{i}$ is contained in $D$. If a colour $d \in D$ is repeated in some $H_{i}$, then it does not occur at all in any other subgraph $H_{j}$ with $j \geqslant 2$ : indeed, a vertex $v$ in $H_{j}$ with colour $d$ would be joined to at least one of the vertices coloured $d$ in $H_{i}$ by a positive edge, which is impossible. Thus each $H_{i}$ with $i \geqslant 2$ has a different repeated colour from $D$. However, there are $n-1$ all-negative subgraphs $H_{i}$ in $G_{n}$ while there are only $n-2$ elements in $D$, proving that a colouring of $G_{n}$ with colours from $M_{2 n-2}$ is not feasible.

A signed graph is antibalanced if the sign product on every even circuit is +1 and the sign product on every odd circuit is -1 . The signature of an antibalanced signed graph is easily seen to be switching equivalent to the all-negative signature. Furthermore, it is well known [6] that a signed graph is antibalanced if and only if its vertex set can be partitioned into two sets (either of which may be empty) such that each edge between the sets is positive and each edge within a set is negative. A balanced antibalanced signed graph is necessarily bipartite, so antibalanced signed graphs are a natural generalisation of bipartite graphs. The next result is an obvious extension of the familiar characterisation of bipartite graphs.

Proposition 2. A signed graph is 2-colourable (that is, $\chi(G) \leqslant 2$ ) if and only if it is antibalanced.

Proof. Let $G$ be a signed graph. If $G$ is antibalanced, we can switch the signature of $G$ to make it all-negative and assign 1 to all the vertices. This produces a 2-colouring of $G$, 
so $\chi(G) \leqslant 2$. For the converse assume that $G$ admits a 2-colouring. The colour classes form a partition into sets $V_{1}$ and $V_{-1}$ which has the property that each edge within the sets is negative and each edge between the sets is positive. By the result of [6] mentioned above, $G$ is antibalanced.

A graph $H$ is called $k$-degenerate if every subgraph of $H$ has a vertex of degree at most $k$. It is well known and easy to see that a graph is $k$-degenerate if and only if there is an ordering $v_{1}, v_{2}, \ldots, v_{n}$ of its vertices such that for every $1<i \leqslant n$ the vertex $v_{i}$ has at most $k$ neighbours in $\left\{v_{1}, \ldots, v_{i-1}\right\}$.

Proposition 3. If a signed graph $G$ is $k$-degenerate, then $\chi(G) \leqslant k+1$.

Proof. Order the vertices of $G$ into a sequence $v_{1}, v_{2}, \ldots, v_{n}$ in such a way that for each $i$ with $1<i \leqslant n$ the vertex $v_{i}$ has at most $k$ neighbours within $\left\{v_{1}, v_{2}, \ldots, v_{i-1}\right\}$. Now, colour the vertices greedily in the described order, that is, at each step assign the vertex $v_{i}$ an available colour with the smallest absolute value. Each coloured neighbour $v$ of $v_{i}$ forbids one colour to $v_{i}$ : the colour of $v$ if the edge $v v_{i}$ is positive, or the negative of this colour if the edge $v v_{i}$ is negative. Hence, having $k+1$ colours from $M_{k+1}$ at our disposal, we can colour each $v_{i}$ and produce a proper $(k+1)$-colouring of $G$.

For the next result we need to assume that the signed graph in question is simple. Recall that the vertex arboricity of a graph $H$, denoted by $a(H)$, is the minimum number of subsets into which $V(H)$ can be partitioned so that each set induces a forest. Similarly, the edge arboricity of a graph $H$, denoted by $a^{\prime}(H)$, is the minimum number of forests into which $E(H)$ can be partitioned; equivalently, it is the minimum number of acyclic subgraphs whose union covers $E(H)$.

Proposition 4. Let $G$ be a simple signed graph. The following statements hold true.

(i) If $G$ is $K_{4}$-minor-free, then $\chi(G) \leqslant 3$.

(ii) If $G$ is a union of two forests (that is, if $a^{\prime}(\underline{G}) \leqslant 2$ ), then $\chi(G) \leqslant 4$.

(iii) If $a(G) \leqslant k$, then $\chi(G) \leqslant 2 k$.

Proof. (i) A graph is $K_{4}$-minor-free if and only if it is series-parallel [3, pp. 172-174]. A series-parallel graph may be turned into $K_{2}$ by a sequence two operations: (1) replacement of a pair of parallel edges with a single edge that connects their common end-vertices, and (2) replacement of a pair of edges incident with a vertex of degree 2 with a single edge. From this description it is easy to see that every simple series-parallel graph is 2-degenerate. The result now follows from Proposition 3.

(ii) Let $F_{1}$ and $F_{2}$ be forests whose union is $G$. Without loss of generality we may assume that the forests are spanning and edge-disjoint. To colour $G$, first switch the signature of $G$ to make $F_{1}$ all-negative. Then assign each vertex of $G$ colour 1 or 2 in such a way that adjacent vertices of $F_{2}$ do not receive the same colour. The end-vertices of each edge in $F_{2}$ are now properly coloured. The same is, true for $F_{1}$ because each edge in 
$F_{1}$ is negative and its end-vertices do not receive opposite colours. Thus we have a proper 4-colouring of $G$.

(iii) Let $\left\{V_{1}, V_{2}, \ldots, V_{k}\right\}$ be a partition of $V(G)$ such that the subgraph $F_{i}$ induced by each $V_{i}$ is a forest. Take an arbitrary forest $F_{i}$ and switch vertices within $F_{i}$ to make it all-negative. Since the forests $F_{1}, F_{2}, \ldots, F_{k}$ are pairwise disjoint, switching in one forest does not interfere with switching in other forests. Thus if we assign colour $i$ to each vertex of $F_{i}$, we obtain a proper $2 k$-colouring of $\bigcup_{i=1}^{k} F_{i}$. Observe that each edge of $G$ joining different forests receives colours with different absolute values, so this colouring is also a proper $2 k$-colouring of the entire $G$. This completes the proof.

The idea from Part (iii) of the previous proof can be slightly improved to prove the following.

Proposition 5. For every simple signed graph $G$ we have

$$
\chi(G) \leqslant \chi_{a}(\underline{G})
$$

where $\chi_{a}(\underline{G})$ denotes the acyclic chromatic number of $\underline{G}$.

Proof. By the definition, the colour classes of an acyclic colouring are disjoint independent sets of vertices and every pair of colour classes induces a forest. To prove the result, first assume that $\chi_{a}(\underline{G})$ is even, say $\chi_{a}(\underline{G})=2 k$. Choose an arbitrary acyclic colouring of $\underline{G}$ with $2 k$ colours from the set $\{1,2, \ldots, 2 k\}$. If we arrange the colours into pairs $\{2 i-1,2 i\}$ for $i \in\{1,2, \ldots, k\}$, then we obtain a partition of $V(G)$ into $k$ sets each inducing a forest. Hence $a(\underline{G}) \leqslant k$, and from Proposition 4 (iii) we get $\chi(G) \leqslant 2 k=\chi_{a}(\underline{G})$. To finish the proof assume that $\chi_{a}(\underline{G})$ is odd, say $\chi_{a}(\underline{G})=2 k+1$. In this case we proceed similarly except that we use one more colour $2 k+1$, and recolour the vertices receiving $2 k+1$ from the acyclic colouring with colour 0 . It is easy to see the result is a proper $(2 k+1)$-colouring of $G$, again implying that $\chi(G) \leqslant 2 k+1=\chi_{a}(\underline{G})$.

\section{A Brooks theorem for signed graphs}

The purpose of this section is to state and prove a signed graph version of one of the most fundamental results on graph colourings, the famous theorem of Brooks [4]. Let $G$ be a signed graph with maximum degree $\Delta$. If we colour $G$ greedily with respect to any ordering of its vertices, then, for each vertex in turn at most $\Delta$ colours are forbidden by its previously coloured neighbours. Therefore $\chi(G) \leqslant \Delta+1$ just as in the unsigned case. The bound is clearly reached whenever $G$ is a balanced complete graph or a balanced odd circuit. Indeed, these two families are familiar from the classical Brooks' theorem as the only connected graphs whose chromatic number reaches the bound $\Delta+1$. In the signed case there is one additional extremal class: even unbalanced circuits. Our main result shows that, among connected simple signed graphs, these three infinite families provide the only signed graphs whose chromatic number reaches the bound $\Delta+1$.

Theorem 6. Let $G$ be a simple connected signed graph. If $G$ is not a balanced complete graph, a balanced odd circuit, or an unbalanced even circuit, then $\chi(G) \leqslant \Delta(G)$. 
Before proving Theorem 6 we need several preparatory results. The first of them concerns the chromatic numbers of signed complete graphs.

Proposition 7. If $G$ is a signed complete graph on $n$ vertices, then $\chi(G) \leqslant n$. Furthermore, $\chi(G)=n$ if and only if $G$ is balanced.

Proof. If $G$ is balanced, then clearly $\chi(G)=n$. In the rest of the proof we therefore assume $G$ to be unbalanced and show that $\chi(G) \leqslant n-1$.

We first consider signed complete graphs of odd order and proceed by induction. If $n=3$, then $G$ is switching equivalent to a circuit of length 3 with all edges negative. Putting 1 to all the vertices defines a 2-colouring of $G$, and the conclusion holds. Assume that $n \geqslant 5$ is odd, say $n=2 d+1$. Since $G$ is unbalanced, there is an unbalanced triangle $T$ in $G$. Pick any two vertices $x$ and $y$ of $G$ that do not belong to $T$, and switch the signature of $G$, if necessary, to make the edge $x y$ negative. The graph $G-\{x, y\}$ is an unbalanced complete graph on $n-2$ vertices, so the induction hypothesis guarantees a colouring of $G-\{x, y\}$ with $n-3$ colours from the set $\{ \pm 1, \pm 2, \ldots, \pm(d-1)\}$. It is now sufficient to assign $d$ to both $x$ and $y$, producing a colouring of $G$ with $n-1$ colours from the set $\{ \pm 1, \pm 2, \ldots, \pm d\}$. Thus $\chi(G) \leqslant n-1$.

For $n$ even, remove a suitable vertex $v$ from $G$ in such way that $G-v$ is unbalanced; it is easy to see that such a vertex always exists. By the previous part, we can colour $G-v$ with $n-2$ colours from the set $\{ \pm 1, \pm 2, \ldots, \pm(n-2) / 2\}$. Assigning 0 to $v$ yields an $(n-1)$-colouring of $G$, so $\chi(G) \leqslant n-1$ again.

We further need two lemmas. The first lemma is a standard tool for colouring graphs greedily. The second lemma is due to Lovász and was crucial in his short proof of Brooks' theorem [7]. Its proof can also be found in Cranston and Rabern [5, Lemma 1].

Lemma 8. The vertices of every connected graph $G$ can be ordered in a sequence $x_{1}$, $x_{2}, \ldots, x_{n}$ in such a way that $x_{n}$ is any preassigned vertex of $G$ and for $i<n$ each vertex $x_{i}$ has a neighbour among $x_{i+1}, x_{i+2}, \ldots, x_{n}$.

Lemma 9. Let $G$ be a 2-connected graph with $\Delta(G) \geqslant 3$ other than a complete graph. Then $G$ contains a pair of vertices $a$ and $b$ at distance 2 such that the graph $G-\{a, b\}$ is connected.

Now we are ready to prove Theorem 6 .

Proof of Theorem 6. If $G$ is an unbalanced complete graph, then the conclusion follows from Proposition 7. The conclusion is also true whenever $G$ is a path, a balanced even circuit, or an unbalanced odd circuit. Thus we may assume that $G$ is a simple connected signed graph of order $n$ with maximum degree $\Delta \geqslant 3$ which is not complete. We distinguish two cases.

Case 1. The graph $G$ is 2-connected. By Lemma 9, $G$ contains a path axb such that $a$ is not adjacent to $b$ and $G-\{a, b\}$ is connected. We switch the signature at $a$ and $b$ in such a way that the edges $a x$ and $b x$ are both positive. Next, we choose an 
ordering $x_{1}, x_{2}, \ldots, x_{n-2}$ of the vertices of $G-\{a, b\}$ as in Lemma 8 with $x_{n-2}=x$. We now start colouring $G$ with colours from $M_{\Delta}$ by assigning colour 1 to both $a$ and $b$. Then we colour the vertices $x_{1}, x_{2}, \ldots, x_{n-3}$ greedily in the given order. Each $x_{i} \neq x$ has a neighbour among its successors in $G-\{a, b\}$, so $x_{i}$ has at most $\Delta-1$ neighbours previously coloured. Each coloured neighbour $w$ forbids one colour to $x_{i}$, so at least one colour from $M_{\Delta}$ is still available for $x_{i}$, and we can proceed up to $x_{n-3}$. At last we colour the vertex $x_{n-2}=x$. All the neighbours of $x$ now have their colours, but since the colours of $a$ and $b$ are the same, one colour from $M_{\Delta}$ is available for $x$. Thus we can complete colouring $G$ with colours from $M_{\Delta}$. This establishes Case 1 .

Case 2. The graph $G$ has a cut-vertex. In this case we proceed by contradiction and assume that $G$ is a signed graph of minimum order that cannot be $\Delta$-coloured. We first derive the following property of $G$.

(*) Each cut-vertex $v$ of $G$ is incident with a bridge vw such that $\operatorname{deg}(v)=\operatorname{deg}(w)=\Delta$. Furthermore, $G-v w$ is $\Delta$-colourable and in every $\Delta$-colouring of $G$-vw both $v$ and $w$ are coloured 0 .

To prove $(*)$, take an arbitrary cut-vertex $v$ of $G$ and let $V_{1}, V_{2}, \ldots, V_{s}$ be the vertex sets of the components of $G-v$; let $G_{i}$ denote the subgraph induced by set $V_{i} \cup\{v\}$. By the minimality of $G$, each $G_{i}$ admits a $\Delta$-colouring $c_{i}$. If a certain subgraph $G_{j}$ has $\operatorname{deg}_{G_{j}}(v) \leqslant \Delta-2$, then we can clearly choose $c_{j}$ to have $c_{j}(v) \neq 0$. Note that any permutation of non-zero colours from $M_{\Delta}$ transforms a proper $\Delta$-colouring into another proper $\Delta$-colouring. Thus if we had $\operatorname{deg}_{G_{i}}(v) \leqslant \Delta-2$ for each $i \in\{1,2, \ldots, s\}$, we could permute the colours within each $G_{i}$ in such a way that $c_{i}(v)=1$ for every $i \in\{1,2, \ldots, s\}$. The colourings $c_{1}, c_{2}, \ldots, c_{s}$ then could be easily combined into a $\Delta$-colouring of the whole of $G$, which would be a contradiction. Therefore there exists a subgraph $G_{j}$ such that $\operatorname{deg}_{G_{j}}(v)>\Delta-2$. This is only possible when $\operatorname{deg}_{G_{j}}(v)=\Delta-1, s=2$, and $v$ is incident with a bridge $v w$. Let $G_{v}$ and $G_{w}$ be the components of $G-v w$ containing $v$ and $w$, respectively. Clearly, each of $G_{v}$ and $G_{w}$ has a $\Delta$-colouring. If one of the bridge-ends, say $w$, had a non-zero colour, we could easily permute the colours within $G_{w}$ and then combine the colourings of $G_{v}$ and $G_{w}$ into a $\Delta$-colouring of the entire $G$. Thus for every $\Delta$-colouring $\phi$ of $G_{v}$ and every $\Delta$-colouring $\psi$ of $G_{v}$ we have $\phi(v)=\psi(w)=0$. This clearly forces $\operatorname{deg}(v)=\operatorname{deg}(w)=\Delta$ (and $\Delta$ odd), and establishes $(*)$.

To finish the proof, let us choose the cut-vertex $v$ of $G$ and the bridge $v w$ in such a way that $G_{v}$ is bridgeless. Take any neighbour $u$ of $v$ in $G_{v}$. If $u$ was a cut-vertex, then by $\left(^{*}\right)$ it would be incident with a bridge of $G$, which is impossible because $G_{v}$ was chosen bridgeless. Therefore $G_{v}-u$ is connected. Lemma 8 now ensures that the vertices of $G_{v}-u$ can be ordered into a sequence $x_{2}, x_{3}, \ldots, x_{m}$ with $x_{m}=v$ in such a way that for $2 \leqslant i<m$ each $x_{i}$ has a neighbour among its successors. If we set $x_{1}=u$, the same becomes true for $1 \leqslant i<m$, because $x_{1}$ is adjacent to $x_{m}$. We now assign 0 to $x_{1}$ and colour the vertices greedily in the indicated order. The result is a $\Delta$-colouring of $G_{v}$ under which $v$ receives a non-zero colour, contradicting $(*)$. This contradiction establishes the theorem. 


\section{Chromatic number of signed planar graphs}

We conclude this article with a brief discussion of chromatic numbers of signed planar graphs. Our main result here is a five colour theorem for signed planar graphs.

Theorem 10. For every simple signed planar graph $G$ one has $\chi(G) \leqslant 5$. Furthermore,

(i) if $G$ is triangle-free, then $\chi(G) \leqslant 4$; and

(ii) if $G$ is has girth at least 5 , then $\chi(G) \leqslant 3$.

Proof. Borodin [1] proved that every planar graph is acyclically 5 -colourable. If we combine this fact with Proposition 5, we obtain $\chi(G) \leqslant \chi_{a}(\underline{G}) \leqslant 5$, as claimed. Next, it is an easy consequence of Euler's formula that every triangle-free planar graph contains a vertex of degree at most 3; hence it is 3-degenerate. The statement (i) now follows from Proposition 3. Finally, to prove the statement (ii) we employ a result of Borodin and Glebov [2] that the vertex set of every planar graph of girth at least 5 has a partition $\{U, W\}$ where $U$ is an independent set and $W$ induces a forest. We take the forest $F$ induced by $W$ and switch the signature of $G$ to make $F$ all-negative. Now we can assign the vertices of $U$ colour 0 and those of $W$ colour 1, producing a proper 3-colouring of $G$.

The four colour theorem implies that the underlying graph of every signed planar graph $G$ can be properly coloured with four colours. It is natural to ask whether it is possible to find a 4-colouring of $G$ that respects the constraints of the signature. To this end, we propose the following conjecture:

Conjecture 11. Every simple signed planar graph $G$ has $\chi(G) \leqslant 4$.

\section{Acknowledgements}

The work of the first and the third author was partially supported from the grant VEGA $1 / 0474 / 15$. The research of the second author has been carried out with financial support from the French State, managed by the French National Research Agency (ANR) in the frame of the "Investments for the future" Programme IdEx Bordeaux - CPU (ANR-10IDEX-03-02).

The authors would like tho thank Gábor Wiener for a stimulating discussion on the topic of this paper and to Thomas Zaslavsky for useful suggestions and encouragement.

\section{References}

[1] O. V. Borodin. On acyclic colorings of planar graphs. Discrete Math. 25:211236,1979 .

[2] O. V. Borodin and A. N. Glebov. On the partition of a planar graph of girth 5 into an empty graph and an acyclic subgraph. Diskretn. Anal. Issled. Oper. Ser. 1 8:34-53, 2001 (in Russian) 
[3] A. Brandstädt, V. B. Le, and J. P. Spinrad, Graph Classes: A Survey, SIAM Monographs on Discrete Mathematics and Applications, Book 3, Society for Industrial and Applied Mathematics, Philadelphia, 1999.

[4] R. L. Brooks. On colouring the nodes of a network. Proc. Cambridge Philos. Soc., Math. Phys. Sci. 37: 194-197, 1941.

[5] D. W. Cranston and L. Rabern. Brooks' Theorem and beyond, arXiv:1403.0479.

[6] F. Harary. On the notion of balance of a signed graph. Michigan Math. J. 2:143-146, 1953-1954; Addendum, ibid., preceding p. 1.

[7] L. Lovász. Three short proofs in graph theory. J. Combin. Theory Ser. B 19:269-271, 1975.

[8] T. Zaslavsky. Signed graphs. Discrete Appl. Math. 4:47-74, 1982; Erratum, ibid. $5: 248,1983$.

[9] T. Zaslavsky. Signed graph coloring. Discrete Math. 39:215-228, 1982.

[10] T. Zaslavsky. Chromatic invariants of signed graphs. Discrete Math. 42:287-312, 1982.

[11] T. Zaslavsky. How colorful the signed graph? Discrete Math. 52:279-284, 1984. 